\title{
Remote Monitoring and Control System for Wisdom Agricultural Greenhouse Based on Internet of Things Technology
}

\author{
Hong-ru $\mathrm{LI}^{1}$, Fu-yin BIAN ${ }^{1 *}$, Shi-hao $\mathrm{GU}^{1}$ and Xiao-Bai $\mathrm{LI}^{2}$ \\ ${ }^{1}$ College of Information Science and Engineering, Northeastern University, Shenyang, \\ China, 110819 \\ ${ }^{2}$ Northeast Yucai School, Shenyang, China, 110001 \\ *bfy0709@163.com
}

Keywords: Remote monitor, Agricultural greenhouse, Internet of things, Intelligent control.

\begin{abstract}
The application of agricultural greenhouses is more and more extensive, while the air temperature and humidity and other environmental factors in greenhouses need to be regulated carefully by people as yet. This article designed a monitoring and control system for greenhouses to adjust the factors automatically, intelligently and remotely. As the major part of the system, the remote monitoring and control platform is designed on $\mathrm{B} / \mathrm{S}$ architecture and could be accessed by browser, makes it possible for users to monitor the greenhouse in distance. Users could obtain the environment information like the video, the environment data and related curves in real time, and the service like early warning and intelligent control provided by the embedded intelligent control system designed based on expert rule base. Mobile client software on smartphone makes it more convenient to realize remote monitoring. As it turns out, the system could reduce the labor and improve the efficiency of management to a great extent.
\end{abstract}

\section{Introduction}

With the continuous improvement of living standards, people's demand for high quality vegetables, fruits and flowers has surged in the last few years. Greenhouse planting technology gets rid of the seasonal and geographical restrictions [1], so that crops planted in greenhouse have more diverse species and usually have high quality and yields with fine regulation of the environment factor, hence crops planted in greenhouse satisfy the market demand in a large degree and directly promote the agricultural economic. Therefore the greenhouse planting technology has been widely applied.

Compared to the natural field, the subtly adjustment of the environment factors in greenhouse such as the air temperature, soil moisture, carbon dioxide concentration, light intensity brings heavy control tasks, while the traditional approach relied on people requires a lot of manpower with low efficiency. Therefore in order to realize the automatic control of greenhouse environment and improve the efficiency of production, establishing greenhouse monitoring system is very necessary [2].In recent years, along with the rapid development of communications technology, computer technology and sensor technology, researchers combine the Internet of Things (IoT) technology and automatic control technology to improve the automatic control level of greenhouses, bring agricultural greenhouses one step closer to the wisdom agriculture. 
The main content of this paper is arranged as follows. The first part introduces the overall architecture of the remote monitoring and control system for wisdom agricultural greenhouses and detailed every module in the system; the second part designed the intelligent control system based on expert rule base; and a summary is made in the last part of this article.

\section{Design of the System}

In this paper, the IoT technology has been applied to the perception of environmental information in greenhouse. Combined with intelligent control technology, this paper has designed the remote monitoring and control system for wisdom agricultural greenhouse based on IoT. Through the analysis of historical environment data and the establishment of expert rule base, the designed system has realized the wisdom decision-making for agricultural activities such as irrigation and fertilization. According to the intelligent control strategy made by the expert rule base, this system can adjust the environmental factor intelligently, ensuring that the condition of the environment in greenhouse is suitable for crops. Users can monitor the greenhouse in distance through the internet using PC or smartphone, so users could handle the potential or emergency situation in time and therefore improve the reliability and efficiency of the system.

The system consists of data acquisition module, IP Camera module, intelligent control cabinet, intelligent communication unit, remote monitoring and control platform, and mobile client application. The architecture is shown in figure 1.

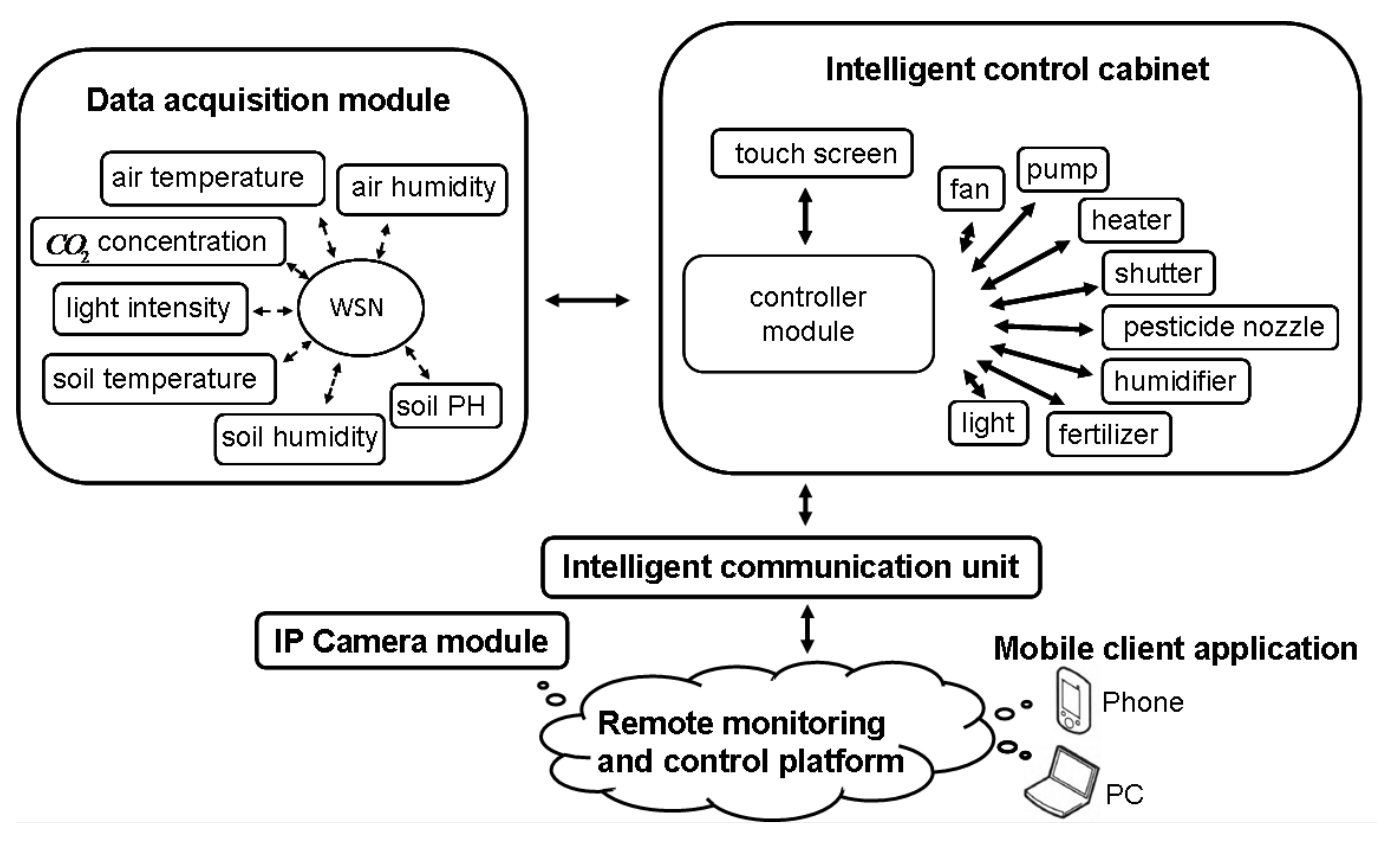

Figure 1. The architecture of the remote monitoring and control system for wisdom agricultural greenhouse based on IoT technology

\section{Data Acquisition Module}

Data acquisition module is mainly composed of various kinds of sensors, such as sensors for air temperature and humidity, soil temperature, soil humidity, concentration of carbon dioxide, light intensity etc. The environmental data collected by all sensors is 
transmitted to the intelligent control cabinet through Wireless Sensor Network (WSN) constructed by ZigBee communication module.

\section{IP Camera Module}

IP Camera module includes an Internet Protocol Camera (IP Camera). It provides numerous benefits such as remote accessibility, two-way audio, infrared night vision and could be connected to the internet directly, thus the local dynamic videos and audio can be transmitted to the cloud server via internet [3].

\section{Intelligent Control Cabinet}

Intelligent control cabinet, act as the control center of all kinds of valves and devices, is composed of controller module, touch screen module, power supply module, etc. Controller module uses chip STM32F105zet6 as the central control unit, and equips with standard digital and analog I/O interface and serial communication interface like RS485 and RS232. On the one hand, this module collects the real-time environmental data of the greenhouse though ZigBee module and RS485 interface and then send them to the intelligent communication unit and the touch screen module. On the other hand, the controller module also controls devices in the greenhouse such as pumps, shutters and fans according to the received control command. Touch screen module includes MCGSTPC - 7062KX, which communicates with the controller module through RS232 interface. This module can display the environmental data and the state of the devices in real time, control the devices by manual and switch to the automatic mode, and alarm when something is abnormal in the whole system.

\section{Intelligent Communication Unit}

Intelligent communication unit can communicate with other parts through RS485 and the internet, its major function is the transmission of data and control commands, making it a hub of communications between the remote monitoring and control platform and the intelligent control cabinet. By establishing communication link with the cloud server, this unit can not only transfer the environmental data to the cloud server but also receive the control commands from the cloud server and send them to the intelligent control cabinet via RS485. Meanwhile, with the $3 \mathrm{G} / 4 \mathrm{G}$ module, the intelligent communication unit could communicate with cloud server wirelessly.

\section{Remote Monitoring and Control Platform}

Based on IoT architecture, using Web servers and database servers as the cloud server, the remote monitoring and control platform for agricultural greenhouse is designed based on B/S (Browser/Server) architecture. The task of the platform is collecting and analyzing all the environment information, scheduling the control task and formulating the control strategy, it makes the platform a data management center of the whole system. The environmental data and the video of the greenhouse are transmitted to the platform through the intelligent control cabinet and the IP Camera module respectively, and then the platform provides users with functions such as displaying, analyzing, early warning and intelligent control, etc. Users access to those services via the internet using the browser, greatly reducing clients' configuration work. With all the data and software on the server side, as well as upgrades and modifications only on the server side, the system is easy to maintain and extend.

Users will be directed to the login page when trying to access the Web server, and then the registered users will be taken to the home page of the software designed for the 
greenhouse remote monitoring and control system. The software is composed of three functions: user management, monitoring function and control strategy management.

The user management includes accounts management and authority management so as to give different users different level of permissions and different application rights.

The monitoring function consists of the following five parts: (1) the device management: add or remove the device in greenhouse such as shutters, fans, water pumps and so on;(2) the node management: plot the topology of WSN, display the information of the node when clicked, for example the location, working state like battery replacement time and duration; (3) display of the states of the greenhouse: display the real-time environmental data of the greenhouse; draw the real time curve and history curve for all the environment factors; display the current state of the device in the greenhouses such as shutters, fans, water pumps and so on; show the video of the greenhouse with modifiable parameters and angles of the camera; as shown in figure 2. (4) alarm management: set up the alarm threshold for the environment factor, customize the alarm condition, alarm by means of software and short message service (SMS), and retrieve the alarm log; (5) report management: generate and print reports automatically from the database according to the requirement of the user.

Control strategy management can be divided into two parts: the regular task and the intelligent control. Immediate task and recurring task could be set in the regular task so that the time duration and other items of the control strategy could be set and carry out. In the meantime, the regular task also provides unified management for a large number of similar tasks, allowing users to start multiple tasks in one setting, as shown in figure 3. The intelligent control is able to automatically identify the growth stage of the crops in the greenhouse, intelligently adjust the environment factors, diagnose and control the disease and pests of the crop, as shown in figure 4 and figure 5.

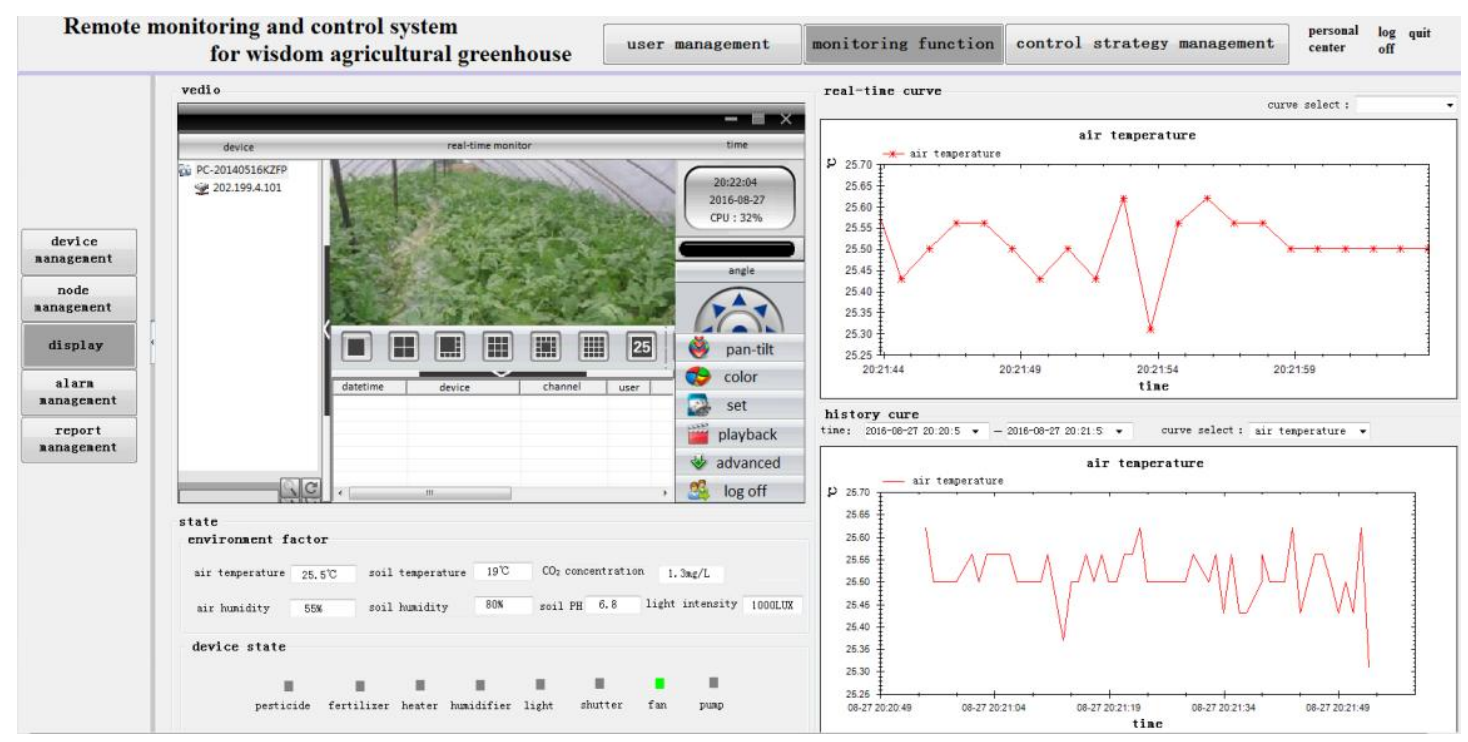

Figure 2.Display of the state of the greenhouse 


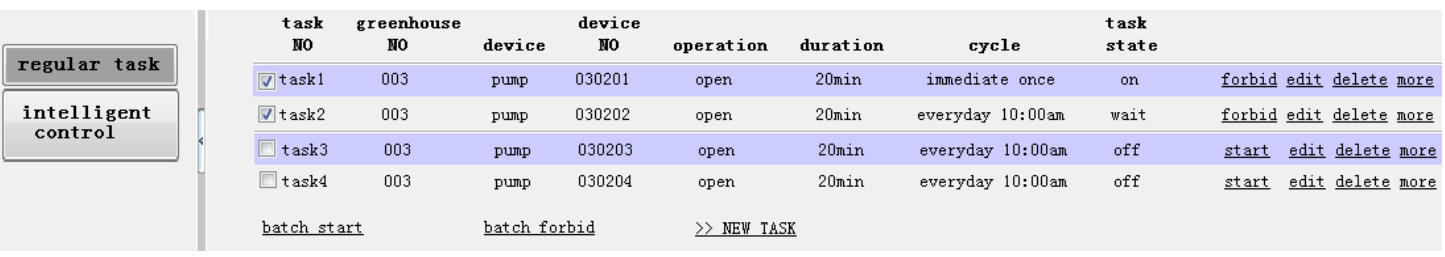

Figure 3. Regular task

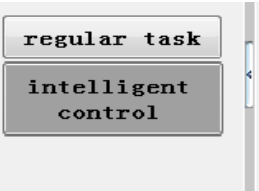

$\square$ intelligently adjust the environment factors

> expert rule base of environment factor intelligent adjustment

V diaqnose and control the disease and pests of the crop

$>$ > expert rule base of diagnose and control the disease and pests

Figure 4. Intelligent control

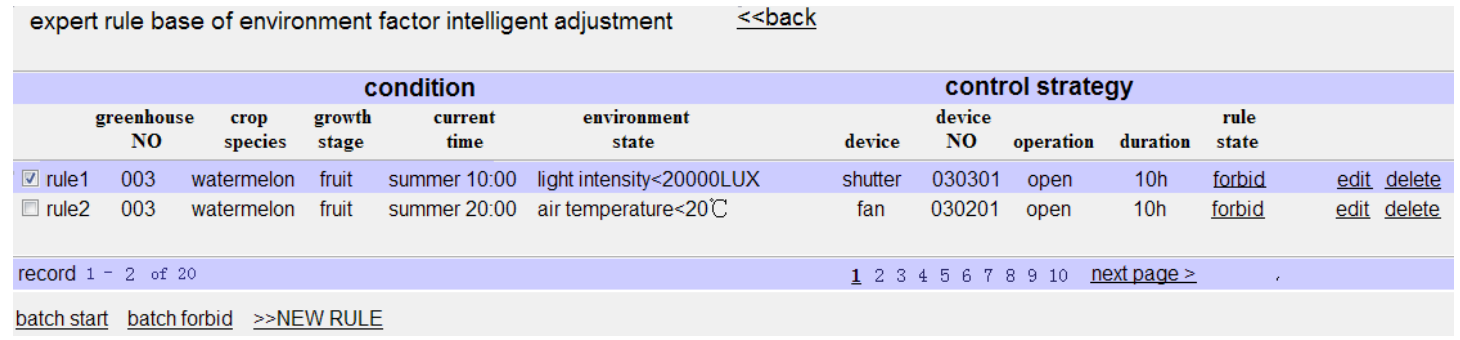

Figure 5. Expert rule base

\section{Mobile Client Application}

In order to remotely monitor the greenhouse more convenient by smartphone, this paper has designed the mobile client application. The application communicates with the cloud server, permitting users to remotely monitor and control the greenhouse anytime and anywhere. The interface of the mobile client application is shown in figure 6 . Users can look over the real-time data of the environment factor and the state of devices in the greenhouse and also can control the devices using the application.
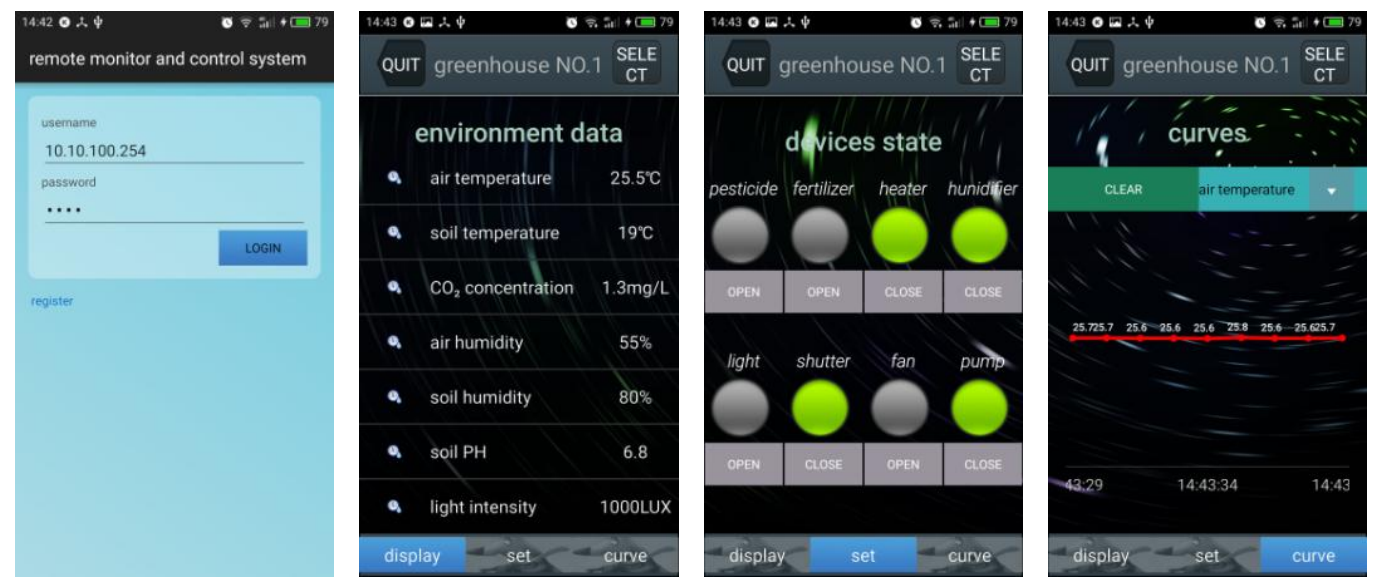

Figure 6. Mobile client application

\section{Design of the Intelligent Control System for Agricultural Greenhouse}

With the environmental data collected by the WSN, such as air temperature, soil humidity, light intensity, as well as the crops data in different growth stages, such as the 
crop growth, yield and quality, the intelligent control system establishes the database of agricultural greenhouses. However, since the environment information of the greenhouse, including images and videos, can be collected at a high frequency automatically and constantly, the data volume grows with astonishing speed [4]. Using big data processing and data mining technology to deal with these data, combining with expert knowledge to analyze the correlation between different attributes, expert rule base can be established. The intelligent control system of agricultural greenhouse implements three functions based on the expert rules base: the automatic recognition of crop growth stages, the intelligent adjustment of environment factors, automatic diagnosing and control of plant diseases and pests.

\section{Crop Growth Stage Recognition System}

Using image processing technology to extract the features of the crop images in different growth stage, and then the crop growth stage model could be set up. The system could recognize the crop growth stage automatically based on the crop growth stage model according to the given crops image in greenhouse.

\section{Environment Factors Intelligent Adjustment System}

In different growth stages and even different moment, there is a big difference in environmental requirements of crops. Taking an example of watermelon in fruiting period, during the day it needs higher light intensity and higher concentration of carbon dioxide to enhance photosynthesis, but at night it needs to lower the temperature and increase the concentration of carbon dioxide to reduce the respiration. Therefore, in order to make the environment more suitable for crop, it is necessary to adjust the light intensity and other environment factors in real time. Based on the analysis of real-time environmental data of the greenhouse and the control rule given by the expert decision-making system, the system transmits control commands to the intelligent control cabinet, controls the devices in greenhouse, and finally realizes intelligent adjustment of environment factors.

\section{Diagnose and Control of Plant Diseases and Pests System}

Image processing technology is utilized to extract the growing situation, leaf color and leaf shape and other features when crop is sick or infected by pests. Combined with the expert experience, the rule base of diagnosing plant diseases and pests is established. By extracting the features of the crop images in greenhouse, and on the basis of the diagnosis rule base, the system could diagnose the crop diseases and insect pests automatically and present preventive measures according to the diagnosis of crop.

\section{Conclusions}

This paper designed a remote monitoring and control system for wisdom agricultural greenhouse. The system realized the real-time acquisition of environment information in greenhouse based on the WSN and the IP Camera, and established the remote monitoring and control platform based on $\mathrm{B} / \mathrm{S}$ mode using the IoT technology and intelligent control technology, and also designed the mobile client application for smartphone. With the design of expert rule base using big data processing and data fusion technology, the system built the agricultural greenhouse intelligent control system. Through PC and smartphones and other remote monitoring terminals, the system designed in this paper realized the remote, real-time and intelligent monitoring and control of agricultural greenhouses. 


\section{Acknowledgement}

This research was financially supported in part by the National Science Foundation of P. R. China Grant (No. 61533007).

\section{References}

[1] Xiaoping Sun, Design and implementation of greenhouse remote monitoring system based on embedded, D. Shenyang University of Technology. 2016. (In Chinese)

[2] Chengnian Zhang: submitted to Xinjiang Farmland Reclamation Science \& Technology, (2016). (In Chinese)

[3] Haochen Ding, Design and implementation of remote visualization intelligent irrigation system based on ARM, D. Dalian University of Technology, 2014. (In Chinese)

[4] Zhongfu Sun, Keming Du, Feixiang Zheng, etc: submitted to Journal of Agricultural Science and Technology, (2013). (In Chinese)

[5] Satyanarayana G V, Mazaruddin S D, Wireless sensor based remote monitoring system for agriculture using ZigBee and GPS, C. Conference on Advances in Communication and Control Systems. (2013) 110-114.

[6] Chang K C, Liu P K, Kuo Z W, et al. Design of persimmon growing stage monitoring system using image recognition technique, C. 2016 IEEE International Conference on Consumer Electronics-Taiwan (ICCE-TW). IEEE, (2016) 1-2.

[7] Soontranon N, Srestasathiern P, Rakwatin P. Rice growing stage monitoring in small-scale region using ExG vegetation index, C. Electrical Engineering/Electronics, Computer, Telecommunications and Information Technology (ECTI-CON), 2014 11th International Conference on. IEEE, (2014) 1-5. 